\title{
A Quantitative Approach for Detecting Symmetries and Complexity in 2D Plane
}

\author{
Mohammad Ali Javaheri Javid ${ }^{1}$, Robert Zimmer ${ }^{1}$, \\ Anna Ursyn ${ }^{2}$, Mohammad Majid al-Rifaie ${ }^{1}$ \\ 1 Department of Computing \\ Goldsmiths, University of London \\ London SE14 6NW, UK \\ $\{$ m.javaheri,r.zimmer,m.majid\}@gold.ac.uk \\ 2 School of Art \& Design \\ University of Northern Colorado \\ Greeley, CO 80639, USA \\ ursyn@unco.edu
}

Keywords: symmetry, complexity, entropy, information gain, cellular automata, 2D patterns

\begin{abstract}
Aesthetic evaluation of computer generated patterns is a growing filed with several challenges. This paper focuses on the quantitative evaluation of order and complexity in multi-state two-dimensional (2D) cellular automata (CA). CA are known for their ability to generate highly complex patterns through simple and well defined local interaction of rules. It is suggested that the order and complexity of $2 \mathrm{D}$ patterns can be quantified by using mean information gain. This measure, also known as conditional entropy, takes into account conditional and joint probabilities of the elements of a configuration in a $2 \mathrm{D}$ plane. A series of experiments is designed to demonstrate the effectiveness of the mean information gain in quantifying the structural order and complexity, including the orientation of symmetries of multi-state 2D CA configurations.
\end{abstract}

\section{Introduction}

The quantitative evaluation of order and complexity of patterns in two dimensional (2D) plane which conforms with human intuitive perception of visual structures is of a great importance in computational aesthetics. Various models have been suggested based on Shannon's information theory to address this problem, however, it is shown that entropic measures fails to discriminate accurately structurally different patterns in $2 \mathrm{D}$ plane $[12,10,9]$.

In this paper, following our previous studies $[12,10,9]$, we examine information gain model, in detecting symmetries, as a measure of order, and randomness of $2 \mathrm{D}$ patterns. We use a multi-state $2 \mathrm{D}$ cellular automaton as our test-bed since they are capable of generating a diverse number of structurally and perceptually distinct $2 \mathrm{D}$ patterns from the iteration of simple rules. Although classical 
one-dimensional cellular automata $(\mathrm{CA})$ with binary states can exhibit complex behaviours, experiments with multi-state 2D CA reveal a very rich spectrum of symmetric and asymmetric patterns $[11,13]$. The main aim of this paper is to demonstrate the effectiveness of information gain as a measure of order and complexity in a $2 \mathrm{D}$ plane.

This paper is organised as follows. Section 2 provides formal definition of CA. Section 3 demonstrates that Shannon entropy is not an adequate measure for evaluating order and complexity in a $2 \mathrm{D}$ plane. Considering human intuitive perception of visual structures, a spatial complexity spectrum is formulated and the potential of information gain as a structural complexity measure is discussed. Section 4 gives details of experiments that examine the effectiveness of information gain. The paper closes with a discussion and results.

\section{Cellular Automata}

CA are one of the early bio-inspired systems invented by von Neumann and Ulam in the late 1940s to study the logic of self-reproduction in a materialindependent framework. CA are known to exhibit complex behaviour from the iterative application of simple rules. The popularity of Conway's Game of Life [8] drew the attention of a wider community of researchers and digital artists to the unexplored potential of CA applications and especially in their capacity to generate complex behaviour [5], often with aesthetic qualities [20].

Definition 1. A cellular automaton is a regular tiling of a lattice with uniform deterministic finite state automata.

A cellular automaton $\mathcal{A}$ is specified by a quadruple $\langle L, S, N, f\rangle$ where:

1. $L$ is a finite square lattice of cells $(i, j)$.

2. $S=\{1,2, \ldots, k\}$ is set of states. Each cell $(i, j)$ in $L$ has a state $s \in S$.

3. $N$ is neighbourhood, as specified by a set of lattice vectors $\left\{e_{a}\right\}, a=$ $1,2, \ldots, N$. The neighbourhood of cell $r=(i, j)$ is $\left\{r+e_{1}, r+e_{2}, \ldots, r+e_{N}\right\}$. A a cell is considered to be in its own neighbourhood so that one of $\left\{e_{a}\right\}$ is the zero vector $(0,0)$. With an economy of notation, the cells in the neighbourhood of $(i, j)$ can be numbered from 1 to $N$; the neighbourhood states of $(i, j)$ can therefore be denoted $\left(s_{1}, s_{2}, \ldots, s_{N}\right)$. Periodic boundary conditions are applied at the edges of the lattice so that complete neighbourhoods exist for every cell in $L$.

4. $f$ is the update rule. $f$ computes the state $s_{1}(t+1)$ of a given cell from the states $\left(s_{1}, s_{2}, \ldots, s_{N}\right)$ of cells in its neighbourhood: $s_{1}(t+1)=f\left(s_{1}, s_{2}, \ldots, s_{N}\right)$. A quiescent state $s_{q}$ satisfies $f\left(s_{q}, s_{q}, \ldots, s_{q}\right)=s_{q}$.

There are two common neighbourhoods; (1) a five-cell von Neumann neighbourhood $\{(0,0),( \pm 1,0),(0, \pm 1)\}$ and $(2)$ a nine-cell Moore neighbourhood $\{(0,0),( \pm 1,0),(0, \pm 1),( \pm 1, \pm 1)\}$. The collection of states for all cells in $L$ is known as a configuration $(C)$. The global rule $F$ maps the whole automaton 
forward in time; it is the synchronous application of $f$ to each cell. The behaviour of a particular $\mathcal{A}$ is the sequence $c^{0}, c^{1}, c^{2}, \ldots, c^{T-1}$, where $c^{0}$ is the initial configuration (IC) at $t=0$.

CA behaviour are sensitive to the IC and to $L, S, N$ and $f$. The behaviour is generally nonlinear and sometimes very complex; no single mathematical analysis can describe, or even estimate, the behaviour of an arbitrary cellular automaton. The vast size of the rule space, and the fact that this rule space is unstructured, mean that knowledge of the behaviour a particular cellular automaton, or even of a set of CA, gives no insight into the behaviour of any other CA. In the lack of any practical model to predict the behaviour of a cellular automaton, the only feasible method is to run simulations.

\section{Measuring Order and Complexity in 2D}

The introduction of information theory provided a quantitative model to measure the order and complexity of systems. Shannon's information theory was an attempt to address reliable communication over an unreliable channel [17]. Entropy is the core of this theory [6]. Let $\mathcal{X}$ be discrete alphabet, $X$ a discrete random variable, $x \in \mathcal{X}$ a particular value of $X$ and $P(x)$ the probability of $x$. Then the entropy, $H(X)$, is:

$$
H(X)=-\sum_{x \in \mathcal{X}} P(x) \log _{2} P(x) .
$$

The quantity $H$ is the average uncertainty in bits, $\log _{2}\left(\frac{1}{p}\right)$ associated with $X$. Entropy can also be interpreted as the average amount of information needed to describe $X$. The value of entropy is always non-negative and reaches its maximum for the uniform distribution, $\log _{2}(|\mathcal{X}|)$ :

$$
0 \leqslant H \leqslant \log _{2}(|\mathcal{X}|)
$$

The lower bound of relation (2) corresponds to a deterministic variable (no uncertainty) and the upper bound corresponds to a maximum uncertainty associated with a random variable. Another interpretation of entropy is as a measure of order and complexity. A low entropy implies low uncertainty so the message is highly predictable, ordered and less complex. And high entropy implies a high uncertainty, less predictability, highly disordered and complex.

Moles [15], Bense [4] and Arnheim [2] were pioneers of the application of entropy to quantify order and complexity by adapting statistical measure of information in aesthetic objects. Since then entropy is commonly used to measure order and complexity in most of aesthetic evaluation functions $[7,14,18,16]$, however entropy fails to discriminate accurately structurally different patterns in two-dimensions. The main reason for this drawback is that it only reflects on the distribution of the symbols, and not on their ordering $[12,10,9]$.

This fact is illustrated in Fig. 1 where the entropy of 2D patterns with different structural characteristics is evaluated. Both of the patterns have a lattice 
size of $18 \times 18$ consisting of three elements (white, blue and orange). The distribution of elements are equally probable $\left(\frac{1}{108}\right)$. Fig. 1a is a pattern with ordered structure, a complete symmetry and Fig. 1b is a fairly structureless (random) pattern.

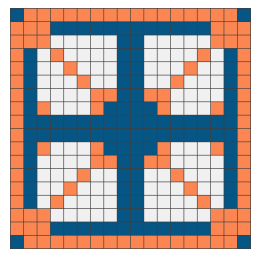

(a)

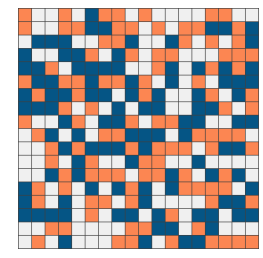

(b)

$H=1.58496$

$$
H=1.58496
$$

Fig. 1. Measurements of $H$ for ordered and random 2D patterns with equally probable distribution of elements.

As it is clear from the comparison of the above patterns with their corresponding entropy value, despite their structural differences, both of the patterns have the same entropy value. This is in contrast to our intuitive perception of the order and complexity of patterns in 2D plane.

If the human perception of visual structures are taken into account in perceiving order and complexity in a 2D plane, any potential measure of order and complexity must be bounded by two extreme points of complete order and disorder. It is reasonable to assume that regular structures, irregular structures and structureless patterns lie along between these extremes, as illustrated in Fig. 2.

$$
\text { order } \stackrel{\text { regular structure } \mid \text { irregular structure } \mid \text { structureless }}{\longleftrightarrow} \text { disorder }
$$

Fig. 2. The spectrum of spatial complexity.

A complete regular structure is a pattern of full symmetry, an irregular structure is a pattern with some local structural orders but not as regular as a fully symmetrical pattern and finally a structureless pattern is a random arrangement of elements.

A measure introduced in $[3,19,1]$ and known as information gain, has been suggested as a means of characterising the complexity of dynamical systems and of patterns in $2 \mathrm{D}$ plane. It measures the amount of information gained in bits when specifying the value, $x$, of a random variable $X$ given knowledge of the value, $y$, of another random variable $Y$,

$$
G_{x, y}=-\log _{2} P(x \mid y) .
$$


$P(x \mid y)$ is the conditional probability of a state $x$ conditioned on the state $y$. Then the mean information gain, $\bar{G}_{X, Y}$, is the average amount of information gain from the description of the all possible states of $Y$ :

$$
\bar{G}_{X, Y}=\sum_{x, y} P(x, y) G_{x, y}=-\sum_{x, y} P(x, y) \log _{2} P(x \mid y)
$$

where $P(x, y)$ is the joint probability, $\operatorname{prob}(X=x, Y=y) . \bar{G}$ is also known as the conditional entropy, $H(X \mid Y)[6]$. Conditional entropy is the reduction in uncertainty of the joint distribution of $X$ and $Y$ given knowledge of $Y, H(X \mid Y)=$ $H(X, Y)-H(Y)$. The lower and upper bounds of $\bar{G}_{X, Y}$ are

$$
0 \leqslant \bar{G}_{X, Y} \leqslant \log _{2}|\mathcal{X}|
$$

Definition 2. A structural complexity measure $G$, of a cellular automaton configuration is the sum of the mean information gains of cells having homogeneous/heterogeneous neighbouring cells over $2 D$ lattice.

For a cellular automaton configuration, $\bar{G}$ can be calculated by considering the distribution of cell states over pairs of cells $r, s$,

$$
\bar{G}_{r, s}=-\sum_{s_{r}, s_{s}} P\left(s_{r}, s_{s}\right) \log _{2} P\left(s_{r}, s_{s}\right)
$$

where $s_{r}, s_{s}$ are the states at $r$ and $s$. Since $|\mathcal{S}|=N, \bar{G}_{r, s}$ is a value in $[0, N]$.

The vertical, horizontal, primary diagonal $(\backslash)$ and secondary diagonal $(\nearrow)$ neighbouring pairs provide eight $\bar{G} s ; \bar{G}_{(i, j),(i-1, j+1)}, \bar{G}_{(i, j),(i, j+1)}, \bar{G}_{(i, j),(i+1, j+1)}$, $\bar{G}_{(i, j),(i-1, j)}, \bar{G}_{(i, j),(i+1, j)}, \bar{G}_{(i, j),(i-1, j-1)}, \bar{G}_{(i, j),(i, j-1)}$ and $\bar{G}_{(i, j),(i+1, j-1)}$.

The relative positions for non-edge cells are given by matrix $M$ :

$$
M=\left[\begin{array}{ccc}
(i-1, j+1) & (i, j+1) & (i+1, j+1) \\
(i-1, j) & (i, j) & (i+1, j) \\
(i-1, j-1) & (i, j-1) & (i+1, j-1)
\end{array}\right] .
$$

Correlations between cells on opposing lattice edges are not considered. The result of this edge condition is that $G_{i+1, j}$ is not necessarily equal to $\bar{G}_{i-1, j}$. In addition the differences between the horizontal (vertical) and two diagonal mean information rates reveal left/right (up/down), primary and secondary diagonals of $2 \mathrm{D}$ patterns. So the sequence of generated configurations by a multi-state $2 \mathrm{D}$ cellular automaton can be analysed by the differences between the vertical $(i, j \pm 1)$, horizontal $(i \pm 1, j)$, primary diagonal $\left(P_{d}\right)$ and secondary diagonal $\left(S_{d}\right)$ mean information gains by

$$
\begin{gathered}
\Delta \bar{G}_{i, j \pm 1}=\left|\bar{G}_{i, j+1}-\bar{G}_{i, j-1}\right|, \\
\Delta \bar{G}_{i \pm 1, j}=\left|\bar{G}_{i-1, j}-\bar{G}_{i+1, j}\right|, \\
\Delta \bar{G}_{P_{d}}=\left|\bar{G}_{i-1, j+1}-\bar{G}_{i+1, j-1}\right|,
\end{gathered}
$$




$$
\Delta \bar{G}_{S_{d}}=\left|\bar{G}_{i+1, j+1}-\bar{G}_{i-1, j-1}\right| .
$$

The mean information gains of the sample patterns in Fig. 1 are presented in Fig. 3. The merits of $\Delta \bar{G} s$ in discriminating structurally different patterns, the full symmetrical (Fig. 3a), the structureless and random (Fig. 3b), are clearly evident.

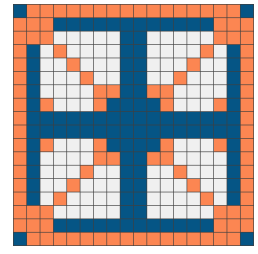

(a)

$H=1.58496$

$\Delta \bar{G}_{i, j \pm 1}=0$

$\Delta \bar{G}_{i \pm 1, j}=0$

$\Delta \bar{G}_{P_{d}}=0$

$\Delta \bar{G}_{S_{d}}=0$

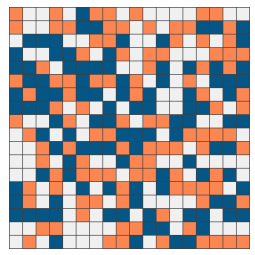

(b)

$$
H=1.58496
$$

$\Delta \bar{G}_{i, j \pm 1}=0.00078$

$\Delta \bar{G}_{i \pm 1, j}=0.00009$

$\Delta \bar{G}_{P_{d}}=0.00052$

$\Delta \bar{G}_{S_{d}}=0.00109$

Fig. 3. The comparison of $H$ with $\Delta \bar{G} s$ for ordered and random $2 \mathrm{D}$ patterns with equally probable distribution of elements.

\section{Experiments and Results}

A series of experiments was designed to investigate the effectiveness of $\Delta \bar{G} s$ in quantifying order (i.e. symmetry) and complexity of patterns generated by a multi-state 2D cellular automaton. A cellular automaton considered for the purpose of experimentations is specified in Table 1. The update rule maps four states, represented by red, blue, orange and white; the quiescent state is white.

The experiments are conducted with three different ICs: (1) all white cells except for a single blue cell at the centre of $65 \times 65$ lattice (Fig. 4a), (2) a 6 cell configuration (Fig. 4b) and (3) a random configuration with 2957 white quiescent states, 417 orange, 403 blue and 448 red cells (Fig. 4c). The update rule has been iterated synchronously for 100 successive time steps. The sequence of generated

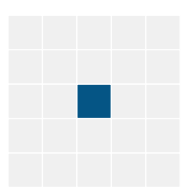

(a)

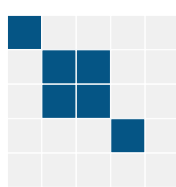

(b)

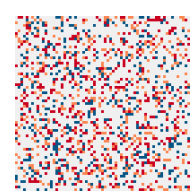

(c)

Fig. 4. The three different ICs. 
Table 1. The update rule of experimental cellular automaton.

$$
\begin{aligned}
& L=65 \times 65 \text { (4225 cells). } \\
& S=\{0,1,2,3\} \equiv\{, \mathbf{\square}, \mathbf{\square}, \mathbf{\square}\} \\
& N \text { : Moore neighbourhood } \\
& f: S^{9} \mapsto S \\
& f\left(s_{i, j}\right)(t)=s_{i, j}(t+1)=\left\{\begin{array}{l}
1 \text { if } s_{(i, j)}(t)=0 \text { and } \sigma=1,3 \\
2 \text { if } s_{(i, j)}(t)=1 \text { and } \sigma=1-8 \\
3 \text { if } s_{(i, j)}(t)=2 \text { and } \sigma=0-8 \\
0 \text { otherwise }
\end{array}\right\}
\end{aligned}
$$

where $\sigma$ is the sum total of the neighbourhood states.

configurations are analysed by Eqs. 8a, 8b, 8d and 8c. Figs. 5,7,9 illustrate the space-time diagrams for a sample of time steps starting from the three ICs.

The behaviour of cellular automaton starting from the single cell IC is a sequence of symmetrical patterns (Fig. 5). The measurements of $\Delta \bar{G} s$ are constant for the 100 time steps $\left(\Delta \bar{G}_{i, j \pm 1}=\Delta \bar{G}_{i, j \pm 1}=\Delta \bar{G}_{P_{d}}=\Delta \bar{G}_{S_{d}}=0\right)$ (Fig. 6). This indicates the development of full symmetrical patterns along the up/down, left/right, primary diagonal and secondary diagonal directions.

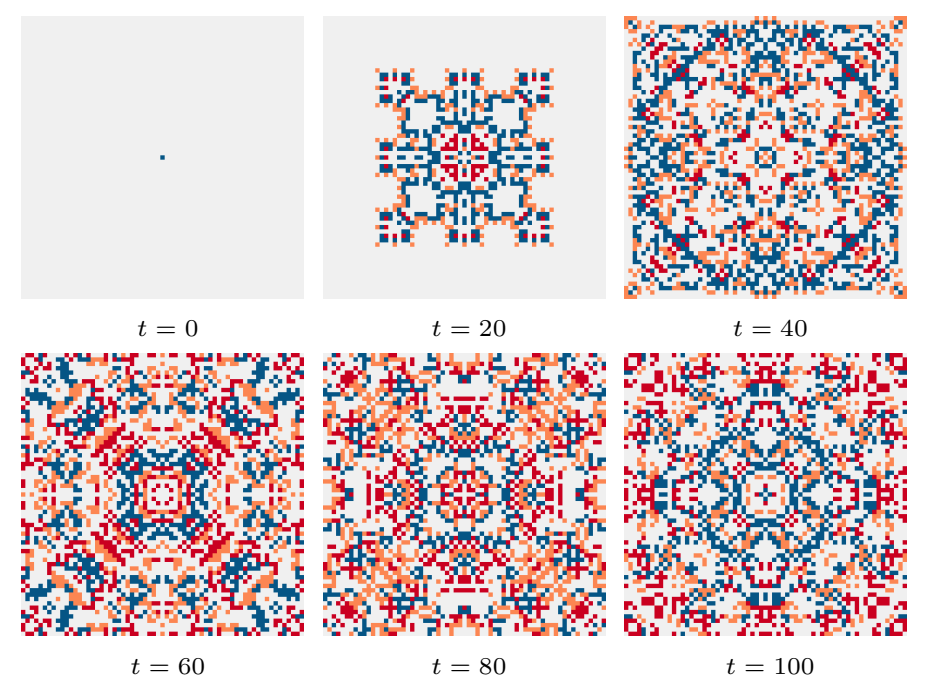

Fig. 5. Space-time diagram of the experimental cellular automaton for sample time steps starting from the single cell IC. 


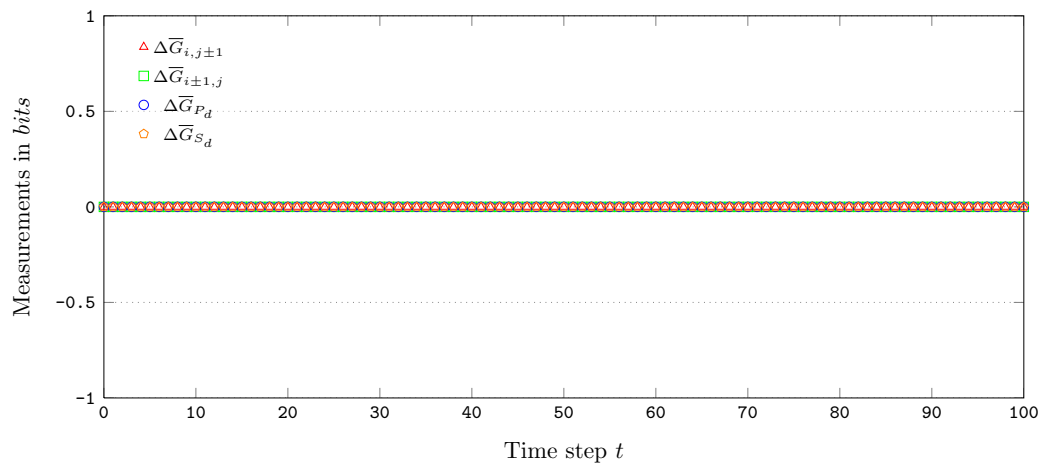

Fig. 6. Measurements of $\Delta \bar{G} s$ for 100 time steps starting from the single IC.

The behaviour of cellular automaton from the 6 cell IC is a sequence of symmetrical patterns with primary diagonal orientations (Fig. 7). The measurements of $\Delta \bar{G} s$, and especially $\Delta \bar{G}_{P_{d}}$ are reflecting the orientation of symmetries (Fig. 8) where $\Delta \bar{G}_{P_{d}}=0$ for 100 time steps.
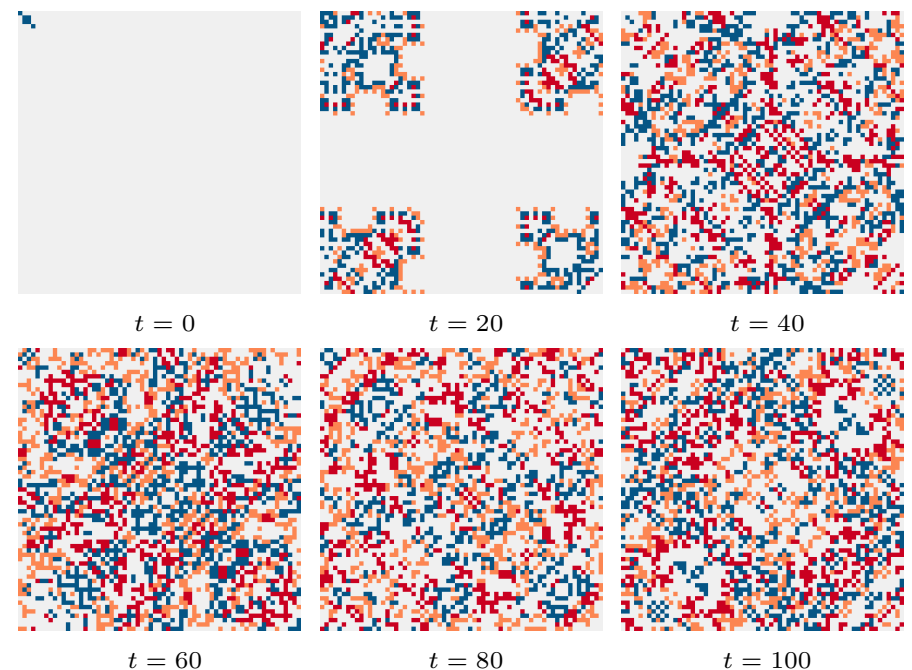

$t=60$

$t=80$

$t=100$

Fig. 7. Space-time diagram of the experimental cellular automaton for sample time steps starting from the 6 cell IC.

The behaviour of cellular automaton starting from the random IC is a sequence of irregular structures with local structures (Fig. 9). $\Delta \bar{G} s$ rates for random IC are plotted in Fig. 10. The measurements of $\Delta \bar{G} s$ are different for all 


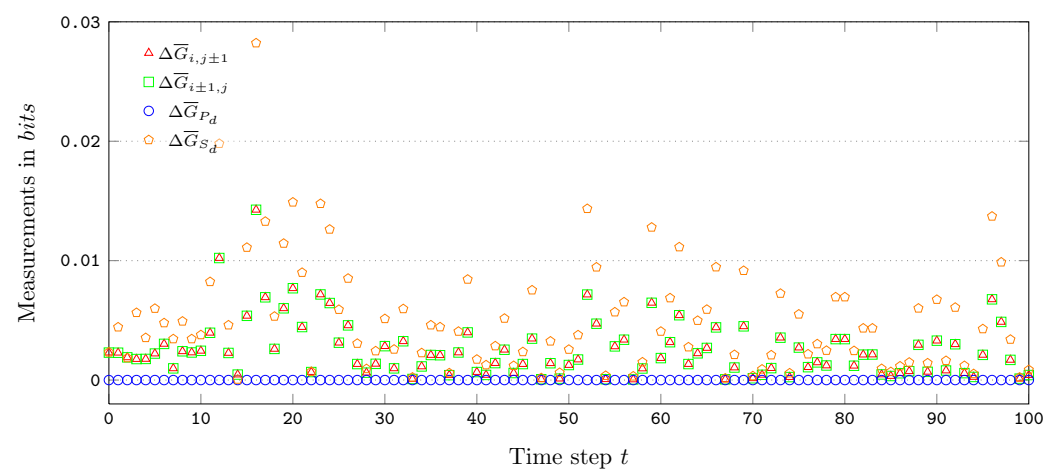

Fig. 8. Measurements of $\Delta \bar{G} s$ for 100 time steps starting from the 6 cell IC.

the directional measurements $\left(\Delta \bar{G}_{i, j \pm 1} \neq \Delta \bar{G}_{i, j \pm 1} \neq \Delta \bar{G}_{P_{d}} \neq \Delta \bar{G}_{S_{d}}\right)$. This is an indicator of the development of irregular structures.

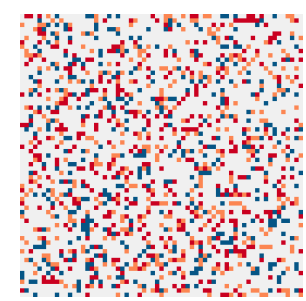

$t=0$

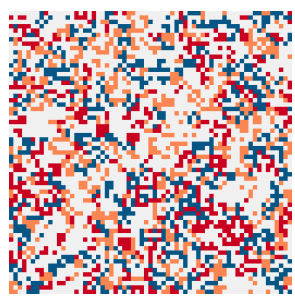

$t=60$

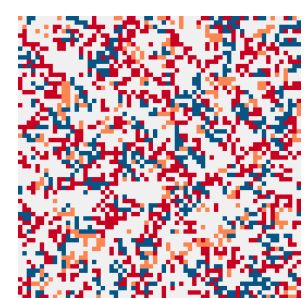

$t=20$

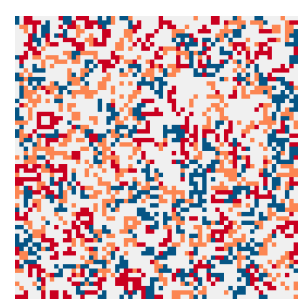

$t=80$

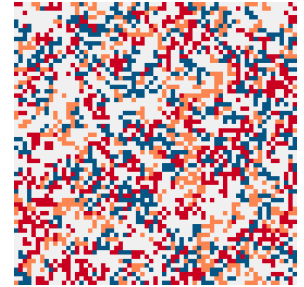

$t=40$

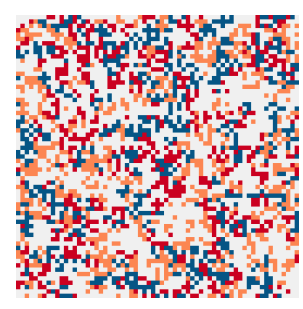

$t=100$

Fig. 9. Space-time diagram of the experimental cellular automaton for sample time steps starting from the random IC (Fig. 4c).

The measurements of $H$ rates for three ICs are plotted in Fig. 11. It is clear that entropy fails to discriminate structural variations in the three set of patterns generated by experimental cellular automaton rule. These experiments demonstrate that a cellular automaton rule seeded with different ICs leads to the formation of patterns with structurally different characteristics. The gradient of the $\Delta \bar{G} s$ rate along lattice axes is able to detect the symmetrical patterns, in- 


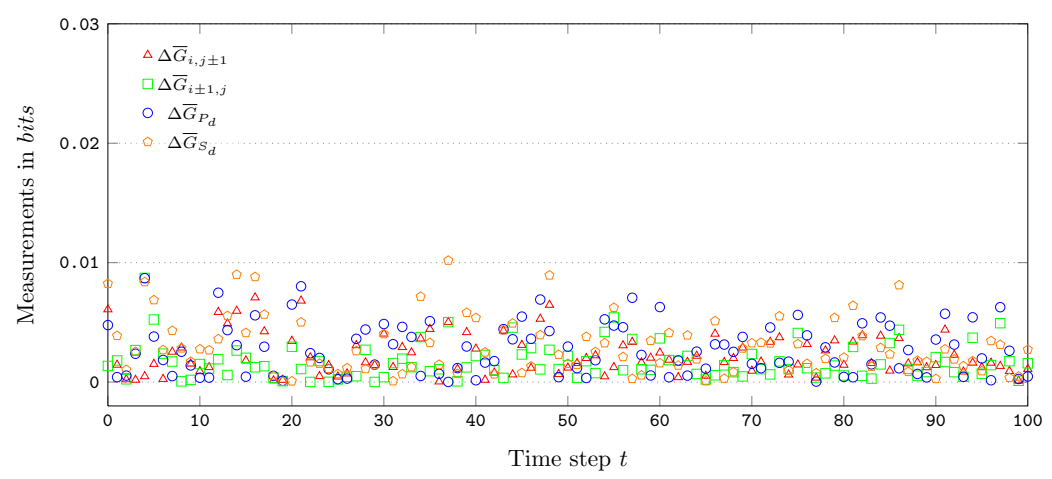

Fig. 10. Measurements of $\Delta \bar{G} s$ for 100 time steps starting from the random IC.

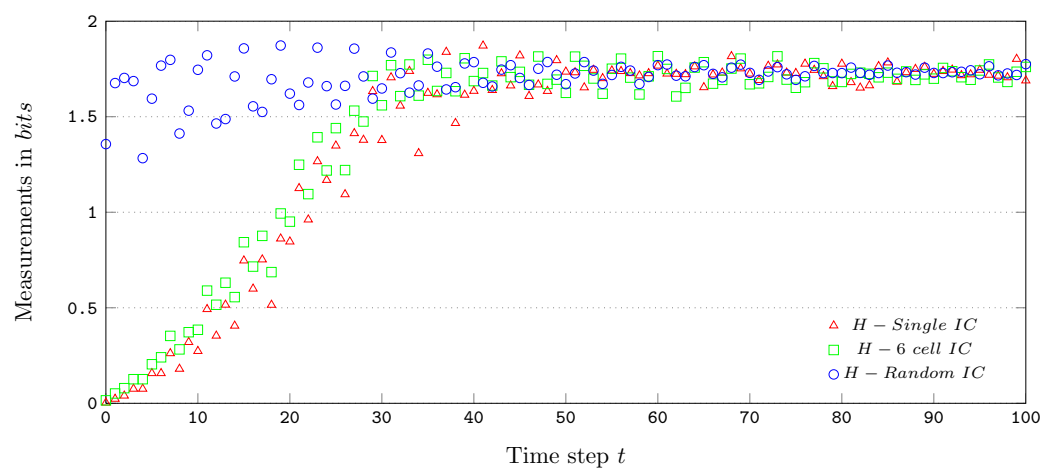

Fig. 11. Measurements of $H$ for 100 time steps starting from the three ICs.

cluding their orientation from the unstructured random configuration generated by this particular multi-state 2D cellular automaton.

\section{Conclusion}

One of the earliest and most well-known bio-inspired models of self-replicating systems is cellular automata (CA). Multi-state two dimensional (2D) CA are capable of generating complex and often aesthetically pleasing configurations. The focus of this work, is an information-theoretic classification of order and complexity in these patterns.

Among the most used statistical measures in the field is entropy which fails to provide a comprehensive picture on the structure of a given input pattern. Mean information gain, on the other hand, is based on correlations between homogeneity and heterogeneity of elements which takes into account conditional and joint probabilities between pairs of elements in 2D plane. Using different initial conditions of a multi-state 2D cellular automaton, this paper presents 
a set experiments to investigate the behaviour of mean information gain and demonstrate its efficiency in distinguishing structurally different configurations. As shown in the paper, mean information gain presents a particularly competitive behaviour in distinguishing symmetries and their orientation.

Acknowledging that CA are one of the generative tools used in computer art, exploring techniques to evaluate the aesthetic qualities of CA generated patterns plays a significant role in enriching the automation of CA art. Furthermore, since information gain measure is based on correlations between homogeneity and heterogeneity of elements, it exhibits a promising application for pattern classification.

\section{References}

1. Andrienko, Yu. A., Brilliantov, N. V., Kurths, J.: Complexity of two-dimensional patterns. Eur. Phys. J. B 15(3), 539-546 (2000)

2. Arnheim, R.: Towards a psychology of art/entropy and art an essay on disorder and order. The Regents of the University of California (1966)

3. Bates, J.E., Shepard, H.K.: Measuring complexity using information fluctuation. Physics Letters A 172(6), 416-425 (1993)

4. Bense, M.: Kleine abstrakte ästhetik [small abstract aesthetics], Edition Rot, vol. 38. E. Walther (March 1969)

5. Brown, P.: Emergent behaviours: Towards computational aesthetics. Artlink 16(2 \& 3) (July 1996)

6. Cover, T.M., Thomas, J.A.: Elements of Information Theory (Wiley Series in Telecommunications and Signal Processing). Wiley-Interscience (2006)

7. Franke, H.W.: A cybernetic approach to aesthetics. Leonardo 10(3), 203-206 (1977)

8. Gardner, M.: Mathematical games - the fantastic combinations of john conway's new solitaire game, life. Scientific American pp. 120-123 (Oct 1970)

9. Javaheri Javid, M.A., Blackwell, T., al Rifaie, M.M., Zimmer, R.: Information Gain Measure for Structural Discrimination of Cellular Automata Configurations. In: 2015 7th Computer Science and Electronic Engineering Conference (CEEC) (CEEC'15). Colchester, Essex, United Kingdom (Sep 2015)

10. Javaheri Javid, M.A., Blackwell, T., Zimmer, R., al Rifaie, M.M.: Spatial Complexity Measure for Characterising Cellular Automata Generated 2D Patterns. In: Pereira, F. and Machado, P. and Costa, E. and Cardoso, A. (ed.) Progress in Artificial Intelligence: 17th Portuguese Conference on Artificial Intelligence, EPIA 2015, Coimbra, Portugal, September 8-11, 2015. Proceedings. Lecture Notes in Artificial Intelligence, vol. 9273, pp. 201-212. Springer International Publishing (2015)

11. Javaheri Javid, M.A., al Rifaie, M.M., Zimmer, R.: Detecting Symmetry in Cellular Automata Generated Patterns Using Swarm Intelligence. In: Dediu, A.H., Lozano, M., Martín-Vide, C. (eds.) Theory and Practice of Natural Computing. Lecture Notes in Computer Science, vol. 8890, pp. 83-94. Springer International Publishing (2014)

12. Javaheri Javid, M.A., al Rifaie, M.M., Zimmer, R.: An Informational Model for Cellular Automata Aesthetic Measure. In: AISB 2015 Symposium on Computational Creativity. University of Kent, Canterbury, UK (2015)

13. Javaheri Javid, M.A., te Boekhorst, R.: Cell Dormancy in Cellular Automata. In: Alexandrov, V.N., van Albada, G.D., Sloot, P.M.A., Dongarra, J. (eds.) International Conference on Computational Science (3). Lecture Notes in Computer Science, vol. 3993, pp. 367-374. Springer (2006) 
14. Machado, P., Cardoso, A.: Computing aesthetics. In: Advances in Artificial Intelligence, pp. 219-228. Springer (1998)

15. Moles, A.: Information theory and esthetic perception. Trans. JE Cohen. U. Illinois Press (1968)

16. Rigau, J., Feixas, M., Sbert, M.: Informational aesthetics measures. Computer Graphics and Applications, IEEE 28(2), 24-34 (2008)

17. Shannon, C.: A Mathematical Theory of Communication. The Bell System Technical Journal 27, 379-423 \& 623-656 (Oct 1948)

18. Staudek, T.: Exact Aesthetics. Object and Scene to Message. Ph.D. thesis, Faculty of Informatics, Masaryk University of Brno (2002)

19. Wackerbauer, R., Witt, A., Atmanspacher, H., Kurths, J., Scheingraber, H.: A comparative classification of complexity measures. Chaos, Solitons \& Fractals 4(1), 133-173 (1994)

20. Wolfram, S.: A New Kind of Science. Wolfram Media Inc. (2002) 